\title{
of paternal and peripubertal stress on aggression, anxiety, and metabolic alterations in the lateral septum
}

\author{
Cordero MI ${ }^{1,2 *}$, Just $\mathbf{N}^{3,4}$, Poirier $\mathbf{G L}^{1}$ and Sandi $\mathrm{C}^{1}$
}

(1) Laboratory of Behavioral Genetics, Brain Mind Institute, Ecole Polytechnique Federale de Lausanne (EPFL), Lausanne, Switzerland

(2) Faculty of Health, Psychology and Social Care. Manchester Metropolitan University. Brooks Building. 53 Bonsall Street. Manchester. United Kingdom.

(3) Animal Imaging and Technology Core, Ecole Polytechnique Fédérale de Lausanne, Lausanne, Switzerland

(4) Department of Radiology, University of Lausanne, Lausanne, Switzerland

\author{
* Corresponding author: \\ Dr. Maria Isabel Cordero \\ Faculty of Health, Psychology and Social Care \\ Manchester Metropolitan University \\ Brooks Building \\ 53 Bonsall Street \\ Manchester \\ M15 6GX \\ United Kingdom
}

Tel.: +44(0)1612472588

m.cordero@mmu.ac.uk

Key Words: Puberty stress, paternal stress, metabolites, lateral septum, aggressive behavior, anxiety

Count:

Abstract: $\quad 252$

Total word-count: 5003 (without references).

Figures: 4

Tables: $\quad 0$

Total references: $\quad 76$

Suppplementary material: 2 figures and 3 tables 


\begin{abstract}
Early-life stress and biological predispositions are linked to mood and personality disorders related to aggressive behavior. We previously showed that exposure to peripubertal stress leads to increased anxiety-like behaviors and aggression against males and females, as well as increased aggression against females in their male offspring. Here, we investigated whether paternal (pS) and individual (iS) exposure to peripubertal stress may exert additive effects on the long-term programming of anxiety-like and aggressive behaviors in rats. Given the key role of the lateral septum (LS) in the regulation of anxiety and aggressive behaviors and the hypothesized alterations in balance between neural excitation and inhibition in aggression-related disorders, markers for these processes were examined in the LS. Peripubertal stress was applied both in naïve male rats and in the offspring of peripubertally stressed males, and anxiety-like and aggressive behaviors were assessed at adulthood. Proton magnetic resonance spectroscopy at 6months, and post-mortem analysis of glutamic acid decarboxylase 67 (GAD67) at 12-months were conducted in LS. We confirmed that aggressive behavior was increased by pS and iS, while only iS increased anxiety-like behavior. Individual stress led to reduced GABA, confirmed by reduced GAD67 immunolabelling, and increased Glutamate, N-acetyl-aspartate, Phosphocholine and Creatine; while pS specifically led to reduced Phosphocreatine. $\mathrm{pS}$ and iS do not interact and exert a differential impact on the analyzed aspects of brain function and anxiety-like behaviors. These data support the view that earlylife stress can affect the behavioral and neurodevelopmental trajectories of individuals and their offspring, which may involve different neurobiological mechanisms.
\end{abstract}




\section{Introduction}

Early-life stress and biological predisposition are both frequently underlined as high-risk etiological factors for the development of pathological aggression. For example, early-life adverse experiences have been shown to increase the risk of violence (Duke et al., 2010), and epidemiological studies have shown a high concordance of aggression and criminal offences among relatives, which suggests that biological predispositions account for an aggression trait (Huesmann et al., 1984). In parallel to findings in humans, we have previously shown that male rats exposed to stress during puberty show an increase in aggressive and anxiety-like behaviors (Marquez et al., 2013; Tzanoulinou et al., 2014a; Tzanoulinou et al., 2014b), with increased aggressive behavior against females also observed in their offspring (Cordero et al., 2012).

Family, twin, and adoption studies suggest that the interaction of biological predisposition and early-life stress increases the risk of developing mental disorders [for review, (Tsuang et al., 2004)]. However, although some studies have found that adopted children of aggressive biological parents have shown a significant increase in aggressive behavior when they were raised in an adverse environment (Cadoret et al., 1995; Caspi et al., 2002; Taylor and Kim-Cohen, 2007), other studies have failed to reproduce these results (Mednick et al., 1984; Willerman et al., 1992). Several factors, including nutrition, exercise, addictions, education, and parental style, are differentially expressed in different human cohorts and may contribute to this discrepancy (Ge et al., 1996; Murray et al., 2012; O'Connor et al., 1998). Thus, using an animal model of peripubertal stress-induced psychopathology that is devoid of some of the limitations intrinsic to human studies, we sought to study whether parental stress would interact with peripubertal stress in the behavioral and neurodevelopmental programing of male rats.

The lateral septum (LS) is one of the critical brain areas that modulates both anxiety and aggression (Albert and Walsh, 1982; Haller et al., 2006; Toth et al., 2010; Veenema and Neumann, 2007). In animals, lesions to the LS increase aggressive behavior (Albert and Walsh, 1982; Clemente and Chase, 1973); moreover, in humans with antisocial personality disorder, those with neurodevelopmental septum abnormalities present higher levels of antisocial personality, psychopathy, and criminal convictions (Raine et al., 2010). The LS is greatly activated during aversive and threatening situations (Duncan et al., 1996) and is involved in the regulation of autonomic (Kubo et al., 2002) and the hypothalamus-pituitaryadrenal (HPA) axis (Herman et al., 2003) responses to stress.

We sought here to investigate the potential neurochemical alterations in the LS in animals exposed to parental and/or individual stress and focused on markers of the balance between neural excitation and inhibition (E/I) and energy metabolism (Bustillo, 2013; Maddock and Buonocore, 2012; Rezin et al., 
2009), because both processes have been hypothesized to be involved in dysfunctions in anxiety and aggression (Herman et al., 2004; Miczek et al., 2007). Currently, the existing neurochemical information related to the $\mathrm{E} / \mathrm{I}$ ratio is scarce. In fact, in humans, this has been frequently provided by proton magnetic resonance spectroscopy ( ${ }^{1} \mathrm{H}$ MRS) obtained from the whole brain (Pollack et al., 2008); although, when focusing on a single brain region (e.g., the anterior cingulate or insula), the measurements are specifically focused on either glutamate (Modi et al., 2014) or GABA (Rosso et al., 2014) levels. Recently, a study reported a correlation between the CSF glutamate levels and impulsive aggression (Coccaro et al., 2013). In animals, the information from microdialysis studies evaluating neurotransmitter levels (SustkovaFiserova et al., 2009) and from protein (Kohl et al., 2013) and gene (Tzanoulinou et al., 2014a; Tzanoulinou et al., 2014b) expression studies that measured markers of glutamatergic and/or GABAergic transmission (e.g., receptors, transporters) in different brain areas further supports the relevance of investigating potential alterations in the neural $\mathrm{E} / \mathrm{I}$ imbalance in the context of stress-induced alterations in anxiety and aggression.

\section{Experimental procedures}

\subsection{Animals}

The experimental subjects were the male offspring (F1) of Wistar Han rats bred in our animal house (F0), which were the progeny of Wistar Han rats purchased from Charles River Laboratories (Lyon, France). The animals were maintained under controlled conditions (12 hr light/dark cycle; lights on at 7:00 AM; $22 \pm 2{ }^{\circ} \mathrm{C}$ ), and food and water available ad libitum. The males (F0 and F1) from different litters were weaned at postnatal day 21 . The male rats from different litters were mixed within each group and were housed at three per standard plastic cage (F0: N=12/group; F1: control father, $\mathrm{N}=26$; F1: stress father, $\mathrm{N}=22$ ). Male and female Wistar Han rats (8 weeks old) purchased from the same supplier were used as intruders and as partners, respectively. All procedures conformed to the Swiss National Institutional Guidelines on Animal Experimentation and were approved through a license from the Swiss Cantonal Veterinary Office Committee for Animal Experimentation.

\subsection{Experimental design}

The experimental design is depicted in Fig. 1. At P28, half of the F0 male rats $(\mathrm{N}=12)$ were submitted to the peripubertal stress condition (see details below), and the F0 control animals $(\mathrm{N}=12)$ were handled on the days that their experimental counterparts were exposed to stress. Except for the routine husbandry procedures, all F0 male rats were left undisturbed from P43 until adulthood (P90). At 3.5 months old, the 
F0 male rats were each separately caged with a 3-month-old virgin female for 21 days. At the end of this period, the males were removed before parturition, and tissue paper was provided to the females to facilitate nest building. With the exception of weekly routine husbandry, the litters and dams remained undisturbed until weaning on P21. Using a similar experimental design, half of the F1 males, which were the offspring of the control $(\mathrm{N}=13)$ and stressed $(\mathrm{N}=11)$ F0 rats, were randomly assigned to peripubertal stress condition. F1 groups: paternal-control \& individual-control, $\mathrm{pC}-\mathrm{iC}, \mathrm{N}=13$; paternal-control \& individual-stress, $\mathrm{pC}-\mathrm{iS}, \mathrm{N}=13$; paternal-stress \& individual-control, $\mathrm{pS}-\mathrm{iC}, \mathrm{N}=11$; and paternal-stress \& individual-stress, pS-iS, N=11). The F1 males were left undisturbed from P43 to P90. At 3 months, the F1 males were submitted to the elevated plus maze (EPM) to study the effects of paternal (pS) and individual (iS) peripubertal stress on adulthood anxiety behavior. At 3.5 months, the F1 males were individually caged together with a virgin female for 21 days (cohabitation period), and the aggressive behavior towards the female was assessed (see details below). Ten days after the cohabitation period started, the resident-intruder test was performed to assess intermale aggressive behavior. Given that during the cohabitation most of the females become pregnant, after 21 days of cohabitation period the females were removed before parturition, and the F1 males were re-housed together with their previous male cagemates in a new fresh home cage. Animals were carefully monitored after rehousing them with previous companions (routine observations of the interactions, physical examination and body weight measures), and no signs of distress, harm or injury were found on them. Two months after the cohabitation period, a randomly selected subset of 6 rats from each group was submitted to ${ }^{1} \mathrm{H}$ MRS to study the potential metabolite alterations within the lateral septum induced by $\mathrm{pS}$ and/or iS. At 12 months old, the rats were taken from their home cage for processing for immunohistochemical labeling of the basal GAD67 levels.

The males used as intruders in the aggression tests and the virgin female rats used as partners were balanced into the experimental groups based on their anxiety-like behaviors and weight and were evaluated one week before the aggression tests or cohabitation period began (Cordero et al., 2012; Marquez et al., 2013).

\subsection{Peripubertal stress}

Peripubertal stress was applied following a previously described protocol (Marquez et al., 2013), see Supplementary Materials for details.

\subsection{Behavioral tests}

Elevated plus maze (EPM). The anxiety-like behaviors were evaluated using the EPM (Pellow et al., 1985). The details are given in the Supplementary Methods. The time spent in the arms, latency to enter the open arm, distance moved and transitions between arms were recorded. An experimenter blind to the 
groups conducted the behavioral scoring of the time spent displaying the following behaviors: rearing, stretching, head-dipping, self-grooming and no movement.

Aggressive behavior towards a female partner. During male-female cohabitation (21 days) the home cage was changed 3 times (once per week) between 1700 and $1900 \mathrm{~h}$. This arousing experience stimulates social interactions and was thus used as the starting time of the 30-min behavioral observations (behavioral scoring details below).

Resident-intruder (RI) test. The rats were subjected to the RI test ten days after the cohabitation period started. The RI test protocol was adapted from (Veenema et al., 2006); for details, see the Supplementary Methods.

The following parameters related to male behavior during the cohabitation and resident behavior during the RI test were scored: number of attacks and abnormal attacks (attacks in vulnerable body parts while the intruder is displaying submissive behavior), lateral threat, offensive upright and keep down. The frequency with which the five behavioral parameters occurred was summarized as the total aggressive behavior. With only some exceptions of superficial scars on a few females, visible wounding was absent in all of the animals.

\section{5. ${ }^{1} \mathrm{H}$ MRS study}

Six 6-month-old rats from each F1 group were randomly selected (average weight $=495 \pm 56 \mathrm{~g}$ ) for ${ }^{1} \mathrm{H}$ MRS. Each rat was individually transported from the animal facility to the animal preparation room in a clean cage. Special care was taken to manipulate each animal gently and quietly during the procedure, and only one rat per cage was scanned in a given day. The scans were performed in a time-balanced way between groups. The animals were placed in a dedicated stereotactic rat holder under continuous isoflurane anesthesia $\left(2 \%\right.$ in $\left.\mathrm{O}_{2}\right)$. The animals' body temperature was monitored continuously using a rectal probe and maintained under physiological temperature $\left(37 \pm 1^{\circ} \mathrm{C}\right)$ using warm circulating water around the animal. After each MR session, the animals were gently put back into the transporting cage. Once they were completely awake, they were returned to their own home cage in the animal facility.

The rats were scanned on an actively shielded $9.4 \mathrm{~T} / 31 \mathrm{~cm}$ bore magnet (Magnex, Varian, Palo Alto, California) with $12-\mathrm{cm}$ gradients $(400 \mathrm{mT} / \mathrm{m}$ in $120 \mu \mathrm{s})$. A quadrature Transmit/Receive $17 \mathrm{~mm}$ surface coil was used. The first and second order shims were adjusted using FAST(EST)MAP (Gruetter and Tkac, 2000) in a 27-45 $\mu \mathrm{l}$ volume placed over the LS (Fig. S2; bregma: $1.7 \mathrm{~mm}$ to $0.8 \mathrm{~mm}$ ) by referencing the Paxinos and Watson atlas (Paxinos and Watson, 1998) and using a 3D-Gradient Echo 
sequence. Localized proton spectroscopy was performed using SPECIAL (Mlynarik et al., 2006). The acquisition time was $40 \mathrm{~min}$.

The in vivo ${ }^{1} \mathrm{H}$ MR spectra were processed using LCModel (Provencher, 1993). The absolute metabolite concentrations were obtained using an unsuppressed water signal as a reference (acquired for each rat). In this study, the concentration of 13 metabolites was quantified using databases of the simulated spectra for the metabolites (Mlynarik et al., 2006). The metabolites used in the basic set for LCModel were GABA, glutamate (Glu), glutamine (Gln), N-acetyl-aspartate (NAA), N-acetyl-aspartate-glutamate (NAAG), creatine (Cr), phosphocreatine (PCr), glucose (GLC), lactose (Lac), glycerophosphocholine (GPC), phosphocholine (PCho), taurine (Tau) and myo-inositol (mI).

\subsection{Immunohistochemistry for GAD67}

The rats were anesthetized with sodium pentobarbital $(60 \mathrm{mg} / \mathrm{kg}$, i.p.) and transcardially perfused via the left ventricle with $0.9 \%$ saline followed by $4 \%$ paraformaldehyde in $0.1 \mathrm{M}$ sodium phosphate buffer (PB), $\mathrm{pH}$ 7.4. After removal from the skull, the brains were post-fixed in the same fixative for $4 \mathrm{hr}$, placed in $0.1 \mathrm{M}$ PB containing $30 \%$ sucrose, frozen on dry ice, and kept at $-80^{\circ} \mathrm{C}$ until sectioning. Coronal sections $(30 \mu \mathrm{m})$ obtained on a cryostat (Leica CM3050 S; Leica Microsystems, Wetzlar, Germany) were preserved in a cryoprotective solution (40\% glycerol and $60 \%$ PBS) and kept at $-20^{\circ} \mathrm{C}$ until further processing. For immunohistochemical processing, the sections were first placed in $0.2 \%$ Triton $\mathrm{X}-100$ in PBS (PBST), pH 7.4, before endogenous peroxidase was blocked using a solution of $0.3 \% \mathrm{H}_{2} \mathrm{O}_{2}$ in PBS. The sections were incubated in a solution containing $10 \%$ donkey serum in PBST and the primary antibody rabbit anti-GAD67 (1:3000; Q99259, Acris Antibody GmbH, Herford, Germany) for 90 min at room temperature, followed by $36 \mathrm{hr}$ at $4{ }^{\circ} \mathrm{C}$, which was interrupted by $2 \mathrm{hr}$ of agitation after an overnight incubation. The sections were then washed $(3 \times 10 \mathrm{~min}$ PBST), incubated in PBST containing the antirabbit secondary antibody (1:1000; Vectastain Kit, Vector Laboratories, Burlingame, CA, USA) for 2 hr, followed by 3x10-min washes in PBST, and then incubated with the avidin-biotinylated peroxidase complex (ABC Kit Vectastain, Vector Laboratories, Burlingame, CA, USA) for 1 hr. After rinsing in 0.1 M PBS, the sections were incubated in a 1:1 dilution of nickel-enhanced diaminobenzadine tetrahydrochloride (DAB; Vector Laboratories, Burlingame, CA, USA) for $5 \mathrm{~min}$. This reaction was terminated by rinsing the tissue in cold 0.1 M PBS. The sections were then mounted onto gelatin-coated slides, dried, dehydrated (2x30 sec ethanol 50\%, $30 \mathrm{sec}$ in ethanol 75\%, $30 \mathrm{sec}$ ethanol 95\%, and 2x2 min in ethanol $100 \%$ ). This process was followed by $2 \times 2$ min incubations in xylene, and finally the sections were coverslipped using DPX. The images were viewed on an Olympus BX51 light microscope (New York, USA), photographed with an Olympus Colorview camera, and stored digitally. The images of the 
septum were taken at 20X magnification. All GAD67-immunoreactive cells were manually counted in the LS and ranged between approximately $1.2 \mathrm{~mm}$ to $-0.8 \mathrm{~mm}$ from the bregma (Paxinos and Watson, 1998). The cell counts were normalized to the area of interest to obtain the cell density (cell number/area, cells per $8.5 \mathrm{~mm}^{2}$ ).

\subsection{Statistical analysis}

The SPSS 17.0 (SPSS, Chicago, IL) statistical package was used for the statistical analyses. The normality and homogeneity of variance of the data were tested, and the adjusted statistics were used as required. The square-root (SQRT) transformation was performed on the following behavioral parameters to produce a normal distribution of data prior to the analyses: offensive behavior frequency for the RI test and attack frequency in the aggression tests. Logarithmic transformation was performed on LS GAD67immunoreactive cell number/area. The normally distributed data were analyzed using two-way ANOVAs (with paternal and individual stress as the between-subject variables). The Kruskal-Wallis statistical analysis of variance for nonparametric data was performed. A $P$ value $<0.05$ was accepted as significant.

\section{Results}

\subsection{Anxiety-like behavior}

Anxiety-like behavior was assessed in the EPM. The detailed statistical results are shown in Table S1. The peripubertally stressed (i.e., individual stress, iS) animals displayed an increase of anxiety-like behavior, as shown by a reduction in the time spent in the open arms (Fig. 2A; $P<0.02$ ) and an increase in the time spent in the closed arms of the maze $(P<0.02)$. Additionally, iS increased the distance travelled in the closed arms $(P<0.05)$ and reduced the distance travelled and velocity in the open arms (distance: $P<0.01$; velocity: $P<0.03)$. No significant effect was found for paternal stress $(\mathrm{pS})$ or for the iSxpS interaction on the distance travelled, velocity or time spent in the center, open arms or closed arms. General locomotor activity, as measured by the frequency of crosses to the center, total velocity and total distance travelled, was not affected by iS, pS or iSxpS (Table S1).

Similar results were obtained with other anxiety/stress-related behaviors displayed in the EPM (Fig. 2B). Compared to the non-stressed rats, the iS rats spent a lower percentage of time head-dipping $(P<0.01)$ and rearing $(P<0.05)$ and a higher percent of time self-grooming $(P<0.01)$. No significant differences were found between groups in stretching or in the time spent without movement (iS, pS and iSxpS, $P>0.05 ; c f$. Table S1).

\subsection{Aggressive behavior}


Aggressive behaviors at adulthood towards a male intruder (RI test) and towards a female partner during the period of cohabitation were assessed; the results and detailed statistical analyses are shown in Fig. 2CF and Table S2. Two-way ANOVA showed a significant increase in resident aggressive behavior during the RI test for iS (but not for $\mathrm{pS}$ or the iSxpS interaction) on i) the frequency of offensive behaviors (Fig. 2C: iS, $P<0.03$ ) and ii) the frequency of attacks (Table S2, iS: $P<0.01$ ).

Furthermore, compared with the $\mathrm{pC}$-iC rats, the $\mathrm{pS}$ and iS rats showed an increase of abnormal aggressive behavior, as shown by i) an increase of attacks towards the intruder displaying submissive behavior (Fig. 2D; Kruskal-Wallis test, $\chi=15.41, P<0.01$; post-hoc comparisons $\mathrm{pC}$-iC $v s$ all, $P<0.05$ ) and ii) an increase of aggressive behavior toward a female partner assessed during the period of cohabitation [Fig. 2E; frequency of offensive behavior, $\mathrm{pS}$ and iSxpS: $P<0.02$, iS: n.s.; and Fig. $2 \mathrm{~F}$, number of attacks toward the females: $\mathrm{pS}$ and iSxpS: $P<0.01$, iS: n.s. Post hoc analyses revealed that compared with $\mathrm{pC}$-iC, all of the other groups increased the frequency of offensive behaviors $(P<0.05)$ and number of attacks toward the female partners $(P<0.01)]$.

The aggressive behavior of the iS and $\mathrm{pS}$ rats was not triggered by an increase in the offensive behavior of the intruder during the RI test, as shown by i) an increased number of attacks performed by the resident compared to the total number of attacks during the encounter [resident attacks + intruder attacks, Fig. S1A: Kruskal-Wallis test, $\chi=10.27, P<0.02$; post hoc comparisons $\mathrm{pC}-\mathrm{iC} v s$ all, $P<0.05)]$, and ii) the increased submissive behavior of the intruders [Fig. S1B; iS, $P<0.02$; iSxpS, $P<0.05$; pS, n.s.; post hoc analyses, pC-iC vs all, $P<0.01]$.

\subsection{LS metabolic profile}

The spectra exhibited a good signal to noise ratio ( $\mathrm{SNR}=42 \pm 5,640$ scans; $1 \mathrm{w}=12 \pm 3 \mathrm{~Hz}$ ) and differences in the metabolites' signals were noticeable (Fig. S2). A total of 13 metabolites were reliably quantified with Cramer-Rao lower bounds under 25\% for GABA and Glc, but they were less than 5\% for NAA and Glu and less than $15 \%$ for Lac. The neurochemical profiles (mean \pm SEM) measured within the LS of the four groups are depicted in Fig. 3. The quantitative results and statistical analysis of the metabolite concentrations are summarized in Table S3. Two-way ANOVA showed a significant increase for iS on several metabolite concentrations in the LS (Table S3, Fig. 3), including NAA, PCho, Cr $(P<0.05)$, and Glu $(P<0.01)$, and a tendency towards significance for Lac $[P<0.062$; observed power (computed using alpha $=0.05)=0.47$, whereas GABA was significantly decreased $(P<0.01)$. A significant $\mathrm{pS}$ effect was found for the PCr concentration $(P<0.05)$, but there was no significant $\mathrm{pS}$ effect for the other metabolites analyzed. No significant iSxpS interaction effect was observed for any of the metabolites analyzed, with 
the exception of a tendency towards significance for the PCr concentrations $[P<0.09$; observed power (computed using alpha $=0.05)=0.4]$.

\subsection{Immunohistochemistry for GAD67}

Given the differences in GABA metabolite at the basal levels found in our experiment, we investigated the protein expression levels of GAD67, as this enzyme produces more than $90 \%$ of GABA in the central nervous system (Asada et al., 1996). Visual inspection of the immunolabeled sections showed that the GAD67-expressing neurons are primarily localized in the dorsal part of the septum (Fig. 4B). Although the intermediate subregion exhibits labeled fibers, it, along with the ventral subregion, is mostly void of immunolabeled cells. Therefore, the cell counts are presented only for the dorsal lateral septum (LSD). The labeled neurons exhibit staining that covers the entire soma, often extending to the dendrites, and represents the cytoplasmic labeling that is expected for GAD67 (Fig. 4C). The number of GAD67-ir cells was decreased in the iS rats (Fig. $4 \mathrm{D}, \mathrm{F}_{1,19}=6.9, P<0.02$ ). Paternal stress failed to reach statistical significance $\left[\mathrm{F}_{1,19}=3.4, P<0.08\right.$; observed power (computed using alpha=0.05) $\left.=0.41\right]$, and no significant effect was found for the iSxpS interaction $\left(\mathrm{F}_{1,19}=0.08\right.$, n.s. $)$.

\section{Discussion}

We studied the possible interaction between paternal (pS) and individual (iS) exposure to pubertal stress on aggressive and anxiety-like behaviors and the metabolic correlates within the lateral septum (LS). Confirming earlier findings, both types of stress paradigms increased aggressive behavior, whereas anxiety-like behavior was only augmented in rats exposed to iS. Furthermore, whereas rats submitted to iS showed a reduction in GABA and increased levels of Glu, NAA, Cr and PCho, the pS rats had reduced $\mathrm{PCr}$ metabolite concentrations. However, there was no interaction of $\mathrm{pS}$ and iS on aggressive or anxietylike behaviors or the metabolic profile of the lateral septum.

In agreement with human (Duke et al., 2010; Huesmann et al., 1984; Wolff and Shi, 2012) and animal (Veenema, 2009) research, as well as our previous study (Cordero et al., 2012; Marquez et al., 2013), both paternal and individual early stress exposure can increase aggressive behavior. The novel question in our study was to investigate whether the exposure to stress during puberty would differentially affect aggressive and anxiety-like behaviors in the offspring of peripubertally stressed male rats. Our results failed to find a synergy between paternal and individual stress in affecting the individuals' responses in these behavioral domains. In addition, the aggressive and anxiety-like behaviors were differentially affected by iS and pS treatments. Rats that were stressed during puberty showed an increase in anxietylike behavior, in accord with a strong body of literature showing that early-life stress increases the risk of developing anxiety disorders (Carr et al., 2013; Ganella and Kim, 2014) and with our own previously 
reported results (Marquez et al., 2013). Conversely, the pS treatment did not affect anxiety-like behavior. Similar to the effects found in the pS non-stressed rats, pubertal (and adult) paternal stress in mice was not shown to alter the offspring's anxiety-like behavior, although evidence was reported for its ability to alter the sperm microRNA content and the offspring's HPA stress reactivity (Rodgers et al., 2013). A lack of concordance between aggression and anxiety has likewise been reported in humans; for instance, comorbid anxiety disorders were shown to be present in almost half of the population of adolescents with conduct disorder (Bartz et al., 2008). Furthermore, animal models used to study abnormal aggressive behavior, such as early maternal separation or repeated defeat in adolescence, show either high or low emotional reactivity, respectively (Haller, 2013; Neumann et al., 2010). Furthermore, corticosterone administration during the peripubertal period was found to increase aggressive behavior without affecting the anxiety-like behavior (Veenit et al., 2013). Taken together, these findings support the view that the brain circuitry and neurobiological mechanisms involved in the regulation of the aggressive behavior and emotion may be differentially affected by different developmental factors (Sandi and Haller, 2015).

Anxiety and mood disorders frequently emerge at puberty (Pattwell et al., 2013) and have been related to both decreased GABA (Mohler, 2012; Tzanoulinou et al., 2014a) and altered glutamatergic transmission (Kugaya and Sanacora, 2005). In agreement with those findings, we found that the iS rats have elevated levels of Glu and low levels of GABA in the LS. This reduction in GABA was further supported by a reduction in GAD67, the enzyme that metabolizes Glu to GABA, and is responsible for the maintenance of the basal tonic GABA levels (Asada et al., 1997) observed in the iS rats. Our results agree with the hypothesis that the effects of stress on brain dysfunction and psychopathology may be the result of a disruption of the GABA/Glu inhibitory/excitatory balance within the brain (Bustillo, 2013). Repeated exposure to stress affects the synthesis, release and reuptake of GABA and Glu, which have important effects on neural activity and excitability [for review see (Joëls et al., 2012)]. In addition to anxiety-like behavior, the iS rats displayed enhanced aggressive behavior. In accord with these results, other treatments that, when they are administered during puberty, have been proven to increase aggressive behavior in adulthood, such as the administration of cocaine (Ricci et al., 2005) or anabolic-androgenic steroids (Grimes et al., 2003), induced a remarkable decrease of GAD65, a second GABA synthesizing enzyme that is rapidly activated under phasic GABA demand, in the LS.

In the iS groups, the NAA and Cr metabolite levels were also increased. NAA is often used as a chemical marker of neuronal and functional integrity, and it is involved in axon-glial signaling, osmoregulation, myelination processes (Maddock and Buonocore, 2012), mitochondrial activity and Glu levels (Bates et al., 1996; Petroff et al., 2002). Cr is involved in buffering the brain's energy demand. Notably, $\mathrm{Cr}$ functions as partial agonist at GABA-A receptors (Almeida et al., 2000) and interacts with NMDA 
receptors (Royes et al., 2008). An increase in the NAA and Cr levels could contribute to increased neural excitation in the iS animals. In addition, the PCho metabolite concentration was also increased in the iS rats. Because PCho is involved in cell membrane density and myelin turnover, the elevations of NAA, Cr and PCho suggest increased neuronal turnover and the activation of energy-dependent synthesis. Interestingly, elevated concentrations of choline and total creatine were reported within the hippocampus of high-functioning autistic patients (Suzuki et al., 2010), a disorder that is often accompanied by comorbid enhanced aggression.

In summary, the metabolic profile of the iS animals (increased levels of Glu, NAA, Cr, PCho and reduced GABA) points to an increase of activity within the LS in the iS groups. Increased aggressive behavior, reduced GAD65 within the LS (Ricci et al., 2005) and increased c-fos expression in the LS, amygdala and other subcortical areas (Knyshevski et al., 2005) has been reported in rats that were repeatedly exposed to cocaine during adolescence. Previously, we have reported that rats stressed during puberty showed a tendency toward increased basal activation in the LS, as observed with $\left[{ }^{14} \mathrm{C}\right] 2$-deoxy-glucose autoradiography, (Marquez et al., 2013) and reduced GAD protein and GAD67 gene expression levels in the anatomically connected amygdala (Tzanoulinou et al., 2014a; Tzanoulinou et al., 2014b). Together, these data support a neurodevelopmental model in which early-life adversity involves the disruption of the frontal-limbic circuitry that regulates aggressive behavior (Marquez et al., 2013). This hypothesis agrees with several human studies that have found increased activity in subcortical brain areas in violent adults with impulsive aggression (Coccaro et al., 2007; Raine et al., 1998).

Regarding the effects of $\mathrm{pS}$ on the metabolite concentrations in the LS, the $\mathrm{pS}$ animals showed a reduction of $\mathrm{PCr}$, metabolite that is considered to serve as stored energy reserves. PCr reduction has been related to mitochondrial dysfunction and stress-related mental disorders. For example, a reduction of PCr (and/or $\mathrm{Cr}$ ) related to mitochondrial dysfunction has been found in stress-related animal models [for review (Allen, 2012)]. Reduced PCr has been found in the frontal region of patients with schizophrenia or bipolar disorder, disorders that are suspected to be related to impaired mitochondrial function [for review (Manji et al., 2012)].

In summary, the different effects of iS and $\mathrm{pS}$ on the metabolite concentrations suggest that iS increases and $\mathrm{pS}$ decreases mitochondrial function in the LS. While it is well known that lesions (Brady and Nauta, 1953; Spiegel et al., 1940) or reversible inactivation (Albert and Richmond, 1976; Albert and Wong, 1978) of the septum results in what is called 'septal rage' state (a state characterized by exaggerated defensive responses and attacks to non-threatening stimuli), that suggests that LS output plays an anxiolytic (inhibitory) role on other brain areas involved in emotion and aggression, other studies suggest 
an anxiogenic role for the LS; e.g., LS injections of agonists of the type 2 corticotropin-releasing factor receptor (Crfr2) results in increased anxiety (Bakshi et al., 2002; Henry et al., 2006; Radulovic et al., 1999). Furthermore, a recent study provided evidence supporting that glutamate released into the LS increases neuronal activity, evokes a delayed release of GABA in the LS and inhibits LS neuronal output (Chee et al., 2015). Therefore, further studies are warranted to reveal the impact of the neurochemical changes observed in the current study in the neural circuit that regulates aggressive behaviors.

The increase of aggressive behavior and the metabolic profile of the $\mathrm{pS}$ animals that suggest a low metabolism of the LS agree with an inhibitory role for the LS in the regulation of aggressive behavior (Albert and Walsh, 1982; Clemente and Chase, 1973; Raine et al., 2010). Our results suggest that paternal aggression and the exposure to stress during puberty affect different neurobiological processes within the LS. Although the iS rats showed alterations in the excitatory/inhibitory balance of the LS and an increase in anxiety-like behavior, the pS alterations in the LS may reflect a reduced energy metabolism, which diminished the inhibitory input of the LS to other brain areas that are involved in the activation of aggressive behavior. Together, our data support the hypothesis that etiological factor-dependent processes (such as environmental factors in relation to biological predispositions and developmental periods) affect different neurobiological mechanisms and the activation of brain patterns involved in the regulation of aggressive behavior (Haller, 2013; Sandi and Haller, 2015). Further studies should investigate whether there is a causal link between the observed neurochemical changes in the LS and the behavioral abnormalities induced by paternal and peripubertal stress. 


\section{References}

Albert, D.J., Richmond, S.E., 1976. Hyperreactivity and aggressiveness following infusion of local anesthetic into the lateral septum or surrounding structures. Behav Biol 18, 211-226.

Albert, D.J., Walsh, M.L., 1982. The inhibitory modulation of agonistic behavior in the rat brain: a review. Neurosci Biobehav Rev 6, 125-143.

Albert, D.J., Wong, R.C., 1978. Hyperreactivity, muricide, and intraspecific aggression in the rat produced by infusion of local anesthetic into the lateral septum or surrounding areas. J Comp Physiol Psychol 92, 1062-1073.

Allen, P.J., 2012. Creatine metabolism and psychiatric disorders: Does creatine supplementation have therapeutic value? Neurosci Biobehav Rev 36, 1442-1462.

Almeida, S., Kempinas, W., Lamano Carvalho, T., 2000. Sexual behavior and fertility of male rats submitted to prolonged immobilization-induced stress. Braz J Med Biol Res. 33, 1105-1109.

Asada, H., Kawamura, Y., Maruyama, K., Kume, H., Ding, R., Ji, F.Y., Kanbara, N., Kuzume, H., Sanbo, M., Yagi, T., Obata, K., 1996. Mice lacking the $65 \mathrm{kDa}$ isoform of glutamic acid decarboxylase (GAD65) maintain normal levels of GAD67 and GABA in their brains but are susceptible to seizures. Biochem Biophys Res Commun 229, 891-895.

Asada, H., Kawamura, Y., Maruyama, K., Kume, H., Ding, R.G., Kanbara, N., Kuzume, H., Sanbo, M., Yagi, T., Obata, K., 1997. Cleft palate and decreased brain gamma-aminobutyric acid in mice lacking the 67-kDa isoform of glutamic acid decarboxylase. Proc Natl Acad Sci U S A 94, 6496-6499.

Bakshi, V.P., Smith-Roe, S., Newman, S.M., Grigoriadis, D.E., Kalin, N.H., 2002. Reduction of stressinduced behavior by antagonism of corticotropin-releasing hormone 2 (CRH2) receptors in lateral septum or CRH1 receptors in amygdala. J Neurosci 22, 2926-2935.

Bartz, J.A., Hollander, E., Inga, D.N.a.R.L., 2008. Oxytocin and experimental therapeutics in autism spectrum disorders, Progress in Brain Research. Elsevier, pp. 451-462.

Bates, T.E., Strangward, M., Keelan, J., Davey, G.P., Munro, P.M., Clark, J.B., 1996. Inhibition of Nacetylaspartate production: implications for $1 \mathrm{H}$ MRS studies in vivo. Neuroreport 7, 1397-1400.

Brady, J.V., Nauta, W.J., 1953. Subcortical mechanisms in emotional behavior: affective changes following septal forebrain lesions in the albino rat. J Comp Physiol Psychol 46, 339-346.

Bustillo, J.R., 2013. Use of proton magnetic resonance spectroscopy in the treatment of psychiatric disorders: a critical update. Dialogues Clin Neurosci 15, 329-337.

Cadoret, R., Yates, W., Troughton, E., Woodworth, G., Stewart, M., 1995. Genetic-environmental interaction in the genesis of aggressivity and conduct disorders. Arch Gen Psychiatry. 52, 916924.

Carr, C.P., Martins, C.M., Stingel, A.M., Lemgruber, V.B., Juruena, M.F., 2013. The role of early life stress in adult psychiatric disorders: a systematic review according to childhood trauma subtypes. J Nerv Ment Dis 201, 1007-1020.

Caspi, A., McClay, J., Moffitt, T.E., Mill, J., Martin, J., Craig, I.W., Taylor, A., Poulton, R., 2002. Role of Genotype in the Cycle of Violence in Maltreated Children. Science 297, 851-854.

Chee, M.J., Arrigoni, E., Maratos-Flier, E., 2015. Melanin-concentrating hormone neurons release glutamate for feedforward inhibition of the lateral septum. J Neurosci 35, 3644-3651.

Clemente, C.D., Chase, M.H., 1973. Neurological substrates of aggressive behavior. Annu Rev Physiol 35, 329-356.

Coccaro, E.F., Lee, R., Vezina, P., 2013. Cerebrospinal fluid glutamate concentration correlates with impulsive aggression in human subjects. J Psychiatr Res 47, 1247-1253.

Coccaro, E.F., McCloskey, M.S., Fitzgerald, D.A., Phan, K.L., 2007. Amygdala and orbitofrontal reactivity to social threat in individuals with impulsive aggression. Biol Psychiatry 62, 168-178. 
Cordero, M.I., Poirier, G.L., Marquez, C., Veenit, V., Fontana, X., Salehi, B., Ansermet, F., Sandi, C., 2012. Evidence for biological roots in the transgenerational transmission of intimate partner violence. Transl Psychiatry 2, e106.

Duke, N.N., Pettingell, S.L., McMorris, B.J., Borowsky, I.W., 2010. Adolescent violence perpetration: associations with multiple types of adverse childhood experiences. Pediatrics 125, 15.

Duncan, G.E., Knapp, D.J., Breese, G.R., 1996. Neuroanatomical characterization of Fos induction in rat behavioral models of anxiety. Brain Research 713, 79-91.

Ganella, D.E., Kim, J.H., 2014. Developmental rodent models of fear and anxiety: From neurobiology to pharmacology. Br J Pharmacol 14, 12643.

Ge, X., Conger, R.D., Cadoret, R.J., Neiderhiser, J.M., Yates, W., Troughton, E., Stewart, M.A., 1996. The developmental interface between nature and nurture: A mutual influence model of child antisocial behavior and parent behaviors. Developmental Psychology 32, 574-589.

Grimes, J.M., Ricci, L.A., Melloni, R.H., Jr., 2003. Glutamic acid decarboxylase (GAD65) immunoreactivity in brains of aggressive, adolescent anabolic steroid-treated hamsters. Horm Behav 44, 271-280.

Gruetter, R., Tkac, I., 2000. Field mapping without reference scan using asymmetric echo-planar techniques. Magn Reson Med 43, 319-323.

Haller, J., 2013. The neurobiology of abnormal manifestations of aggression-A review of hypothalamic mechanisms in cats, rodents, and humans. Brain Research Bulletin 93, 97-109.

Haller, J., TÃ ${ }^{3}$ th, M., Halasz, J., De Boer, S.F., 2006. Patterns of violent aggression-induced brain c-fos expression in male mice selected for aggressiveness. Physiology and Behavior 88, 173-182.

Henry, B., Vale, W., Markou, A., 2006. The effect of lateral septum corticotropin-releasing factor receptor 2 activation on anxiety is modulated by stress. J Neurosci 26, 9142-9152.

Herman, J.P., Figueiredo, H., Mueller, N.K., Ulrich-Lai, Y., Ostrander, M.M., Choi, D.C., Cullinan, W.E., 2003. Central mechanisms of stress integration: hierarchical circuitry controlling hypothalamopituitary-adrenocortical responsiveness. Front Neuroendocrinol 24, 151-180.

Herman, J.P., Mueller, N.K., Figueiredo, H., 2004. Role of GABA and Glutamate Circuitry in HypothalamoPituitary-Adrenocortical Stress Integration. Annals of the New York Academy of Sciences 1018, 35-45.

Huesmann, L.R., Eron, L.D., Leonard, M.M., Walder, L.O., 1984. Stability of aggression over time and generations. Developmental Psychology 20, 1120.

Joëls, M., Sarabdjitsingh, R.A., Karst, H., 2012. Unraveling the Time Domains of Corticosteroid Hormone Influences on Brain Activity: Rapid, Slow, and Chronic Modes. Pharmacological Reviews 64, 901938.

Knyshevski, I., Connor, D.F., Harrison, R.J., Ricci, L.A., Melloni, R.H., Jr., 2005. Persistent activation of select forebrain regions in aggressive, adolescent cocaine-treated hamsters. Behav Brain Res 159, 277-286.

Kohl, C., Riccio, O., Grosse, J., Zanoletti, O., Fournier, C., Schmidt, M.V., Sandi, C., 2013. Hippocampal neuroligin-2 overexpression leads to reduced aggression and inhibited novelty reactivity in rats. PLoS One 8, e56871.

Kubo, T., Kanaya, T., Numakura, H., Okajima, H., Hagiwara, Y., Fukumori, R., 2002. The lateral septal area is involved in mediation of immobilization stress-induced blood pressure increase in rats. Neurosci Lett 318, 25-28.

Kugaya, A., Sanacora, G., 2005. Beyond monoamines: glutamatergic function in mood disorders. CNS Spectr 10, 808-819.

Maddock, R.J., Buonocore, M.H., 2012. MR Spectroscopic Studies of the Brain in Psychiatric Disorders. Curr Top Behav Neurosci 1, 1. 
Manji, H., Kato, T., Di Prospero, N.A., Ness, S., Beal, M.F., Krams, M., Chen, G., 2012. Impaired mitochondrial function in psychiatric disorders. Nat Rev Neurosci 13, 293-307.

Marquez, C., Poirier, G.L., Cordero, M.I., Larsen, M.H., Groner, A., Marquis, J., Magistretti, P.J., Trono, D., Sandi, C., 2013. Peripuberty stress leads to abnormal aggression, altered amygdala and orbitofrontal reactivity and increased prefrontal MAOA gene expression. Transl Psychiatry 15, 144.

Mednick, S.A., Gabrielli, W.F., Jr., Hutchings, B., 1984. Genetic influences in criminal convictions: evidence from an adoption cohort. Science 224, 891-894.

Miczek, K.A., de Almeida, R.M.M., Kravitz, E.A., Rissman, E.F., de Boer, S.F., Raine, A., 2007. Neurobiology of Escalated Aggression and Violence. J. Neurosci. 27, 11803-11806.

Mlynarik, V., Gambarota, G., Frenkel, H., Gruetter, R., 2006. Localized short-echo-time proton MR spectroscopy with full signal-intensity acquisition. Magn Reson Med 56, 965-970.

Modi, S., Rana, P., Kaur, P., Rani, N., Khushu, S., 2014. Glutamate level in anterior cingulate predicts anxiety in healthy humans: a magnetic resonance spectroscopy study. Psychiatry Res 224, 34-41.

Mohler, H., 2012. The GABA system in anxiety and depression and its therapeutic potential. Neuropharmacology 62, 42-53.

Murray, J., Farrington, D.P., Sekol, I., 2012. Children's antisocial behavior, mental health, drug use, and educational performance after parental incarceration: a systematic review and meta-analysis. Psychol Bull 138, 175-210.

Neumann, I.D., Veenema, A.H., Beiderbeck, D.I., 2010. Aggression and anxiety: social context and neurobiological links. Front Behav Neurosci 4, 12.

O'Connor, T.G., Deater-Deckard, K., Fulker, D., Rutter, M., Plomin, R., 1998. Genotype-environment correlations in late childhood and early adolescence: antisocial behavioral problems and coercive parenting. Dev Psychol 34, 970-981.

Pattwell, S.S., Lee, F.S., Casey, B.J., 2013. Fear learning and memory across adolescent development: Hormones and Behavior Special Issue: Puberty and Adolescence. Horm Behav 64, 380-389.

Paxinos, G., Watson, C., 1998. The rat brain in stereotaxic coordinates (Fourth Edition). Academic Press, San Diego.

Pellow, S., Chopin, P., File, S.E., Briley, M., 1985. Validation of open:closed arm entries in an elevated plus-maze as a measure of anxiety in the rat. J Neurosci Methods 14, 149-167.

Petroff, O.A., Errante, L.D., Rothman, D.L., Kim, J.H., Spencer, D.D., 2002. Neuronal and glial metabolite content of the epileptogenic human hippocampus. Ann Neurol 52, 635-642.

Pollack, M.H., Jensen, J.E., Simon, N.M., Kaufman, R.E., Renshaw, P.F., 2008. High-field MRS study of GABA, glutamate and glutamine in social anxiety disorder: response to treatment with levetiracetam. Prog Neuropsychopharmacol Biol Psychiatry 32, 739-743.

Provencher, S.W., 1993. Estimation of metabolite concentrations from localized in vivo proton NMR spectra. Magn Reson Med 30, 672-679.

Radulovic, J., Ruhmann, A., Liepold, T., Spiess, J., 1999. Modulation of learning and anxiety by corticotropin-releasing factor (CRF) and stress: differential roles of CRF receptors 1 and 2. J Neurosci 19, 5016-5025.

Raine, A., Lee, L., Yang, Y., Colletti, P., 2010. Neurodevelopmental marker for limbic maldevelopment in antisocial personality disorder and psychopathy. The British Journal of Psychiatry 197, 186-192.

Raine, A., Meloy, J.R., Bihrle, S., Stoddard, J., LaCasse, L., Buchsbaum, M.S., 1998. Reduced prefrontal and increased subcortical brain functioning assessed using positron emission tomography in predatory and affective murderers. Behav Sci Law 16, 319-332.

Rezin, G.T., Amboni, G., Zugno, A.I., Quevedo, J., Streck, E.L., 2009. Mitochondrial dysfunction and psychiatric disorders. Neurochem Res 34, 1021-1029. 
Ricci, L.A., Grimes, J.M., Knyshevski, I., Melloni, R.H., 2005. Repeated cocaine exposure during adolescence alters glutamic acid decarboxylase-65 (GAD65) immunoreactivity in hamster brain: correlation with offensive aggression. Brain Res 28, 131-138.

Rodgers, A.B., Morgan, C.P., Bronson, S.L., Revello, S., Bale, T.L., 2013. Paternal stress exposure alters sperm microRNA content and reprograms offspring HPA stress axis regulation. J Neurosci 33, 9003-9012.

Rosso, I.M., Weiner, M.R., Crowley, D.J., Silveri, M.M., Rauch, S.L., Jensen, J.E., 2014. Insula and anterior cingulate GABA levels in posttraumatic stress disorder: preliminary findings using magnetic resonance spectroscopy. Depress Anxiety 31, 115-123.

Royes, L.F., Fighera, M.R., Furian, A.F., Oliveira, M.S., Fiorenza, N.G., Ferreira, J., da Silva, A.C., Priel, M.R., Ueda, E.S., Calixto, J.B., Cavalheiro, E.A., Mello, C.F., 2008. Neuromodulatory effect of creatine on extracellular action potentials in rat hippocampus: role of NMDA receptors. Neurochem Int 53, 33-37.

Sandi, C., Haller, J., 2015. Stress and the social brain: behavioural effects and neurobiological mechanisms. Nat Rev Neurosci 16, 290-304.

Spiegel, E.A., Miller, H.R., Oppenheimer, M.J., 1940. Forebrain and rage reactions. J Neurophysiol 3, 538548.

Sustkova-Fiserova, M., Vavrova, J., Krsiak, M., 2009. Brain levels of GABA, glutamate and aspartate in sociable, aggressive and timid mice: an in vivo microdialysis study. Neuro Endocrinol Lett 30, 7984.

Suzuki, K., Nishimura, K., Sugihara, G., Nakamura, K., Tsuchiya, K.J., Matsumoto, K., Takebayashi, K., Isoda, H., Sakahara, H., Sugiyama, T., Tsujii, M., Takei, N., Mori, N., 2010. Metabolite alterations in the hippocampus of high-functioning adult subjects with autism. Int J Neuropsychopharmacol 13, 529-534.

Taylor, A., Kim-Cohen, J., 2007. Meta-analysis of gene-environment interactions in developmental psychopathology. Dev Psychopathol 19, 1029-1037.

Toth, M., Fuzesi, T., Halasz, J., Tulogdi, A., Haller, J., 2010. Neural inputs of the hypothalamic "aggression area" in the rat. Behav Brain Res 215, 7-20.

Tsuang, M.T., Bar, J.L., Stone, W.S., Faraone, S.V., 2004. Gene-environment interactions in mental disorders. World Psychiatry 3, 73-83.

Tzanoulinou, S., García-Mompó, C., Castillo-Gómez, E., Veenit, V., Nacher, J., Sandi, C., 2014a. Long-Term Behavioral Programming Induced by Peripuberty Stress in Rats Is Accompanied by GABAergicRelated Alterations in the Amygdala. PLoS One 9, e94666.

Tzanoulinou, S., Riccio, O., de Boer, M.W., Sandi, C., 2014b. Peripubertal stress-induced behavioral changes are associated with altered expression of genes involved in excitation and inhibition in the amygdala. Transl Psychiatry 4, e410.

Veenema, A.H., 2009. Early life stress, the development of aggression and neuroendocrine and neurobiological correlates: What can we learn from animal models? Frontiers in Neuroendocrinology 30, 497-518.

Veenema, A.H., Blume, A., Niederle, D., Buwalda, B., Neumann, I.D., 2006. Effects of early life stress on adult male aggression and hypothalamic vasopressin and serotonin. Eur J Neurosci 24, 17111720.

Veenema, A.H., Neumann, I.D., 2007. Neurobiological mechanisms of aggression and stress coping: a comparative study in mouse and rat selection lines. Brain Behav Evol 70, 274-285.

Veenit, V., Cordero, M.I., Tzanoulinou, S., Sandi, C., 2013. Increased corticosterone in peripubertal rats leads to long-lasting alterations in social exploration and aggression. Front Behav Neurosci 7, 4. 
Willerman, L., Loehlin, J.C., Horn, J.M., 1992. An adoption and a cross-fostering study of the Minnesota Multiphasic Personality Inventory (MMPI) Psychopathic Deviate Scale. Behav Genet 22, 515-529.

Wolff, N., Shi, J., 2012. Childhood and adult trauma experiences of incarcerated persons and their relationship to adult behavioral health problems and treatment. Int J Environ Res Public Health 9, 1908-1926. 




Figure 1. General experimental design and the sequence of behavioral tests and neurobiological measures performed on F1 male. P: postnatal day 


\section{Anxiety-like behavior, elevated plus maze}

A

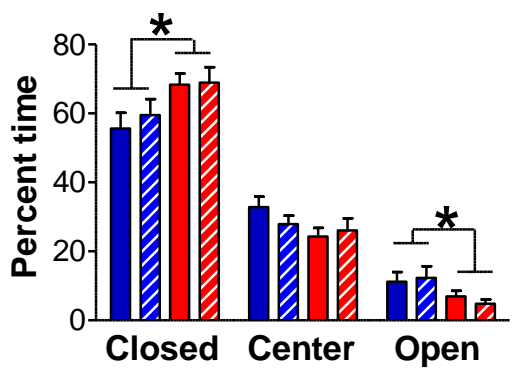

B

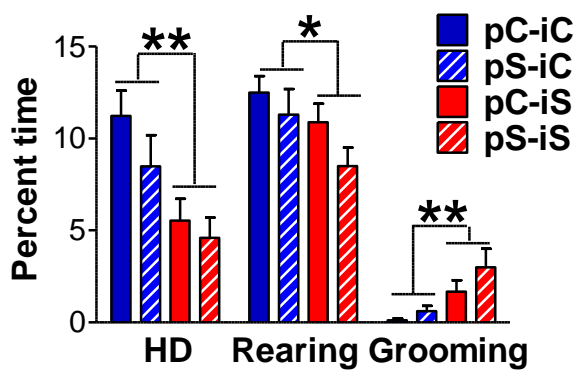

Aggressive behavior, resident-intruder test
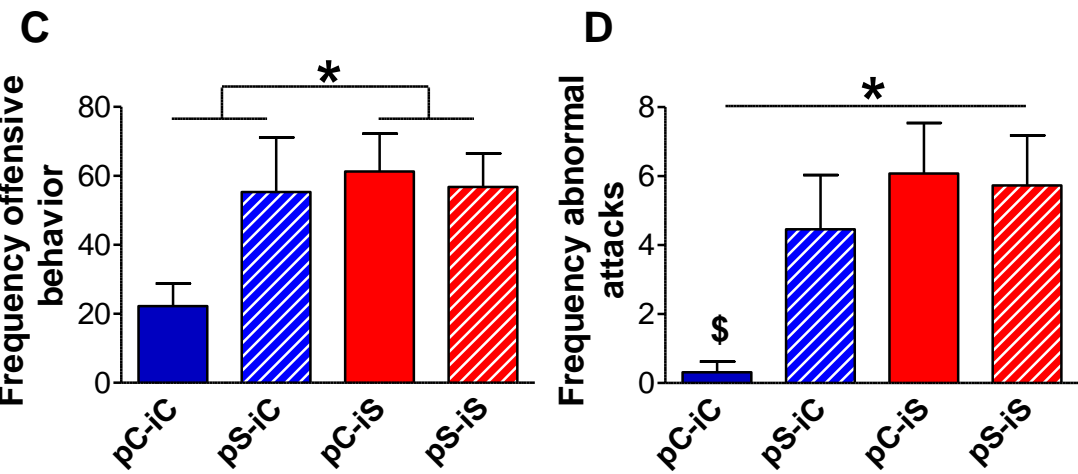

Aggressive behavior towards a female partner

$\mathbf{E}$

$\mathbf{F}$
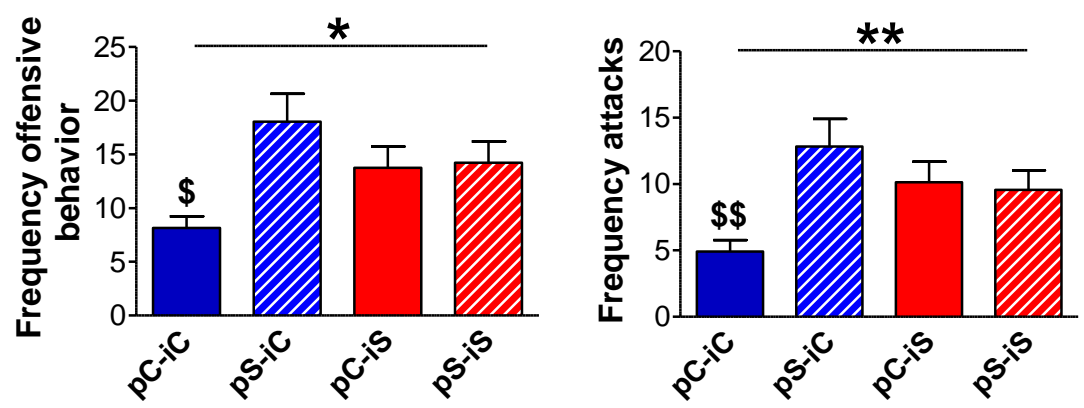

Figure 2. Effects of paternal and individual peripubertal stress on anxiety-like behavior $(A, B)$, aggressive behavior in the resident-intruder test $(C, D)$ and aggressive behavior towards a female partner $(E, F)$ at adulthood. Two-way ANOVA showed a significant effect for individual stress on anxiety-like behavior and aggressive behavior in the resident-intruder test, and for parental stress in aggressive behavior 
towards a female partner. Rats subjected to paternal and/or individual stress showed an increase of aggressive behavior compared to $\mathrm{pC}-\mathrm{iC}$. The results are the mean + SEM. $N=13-11 /$ group. ${ }^{*}, P<0.05 ;{ }^{* *}$, $P<0.01$; Post-hoc analyses: $\$, P<0.05$ vs all, $\$ P<0.01$ vs all.

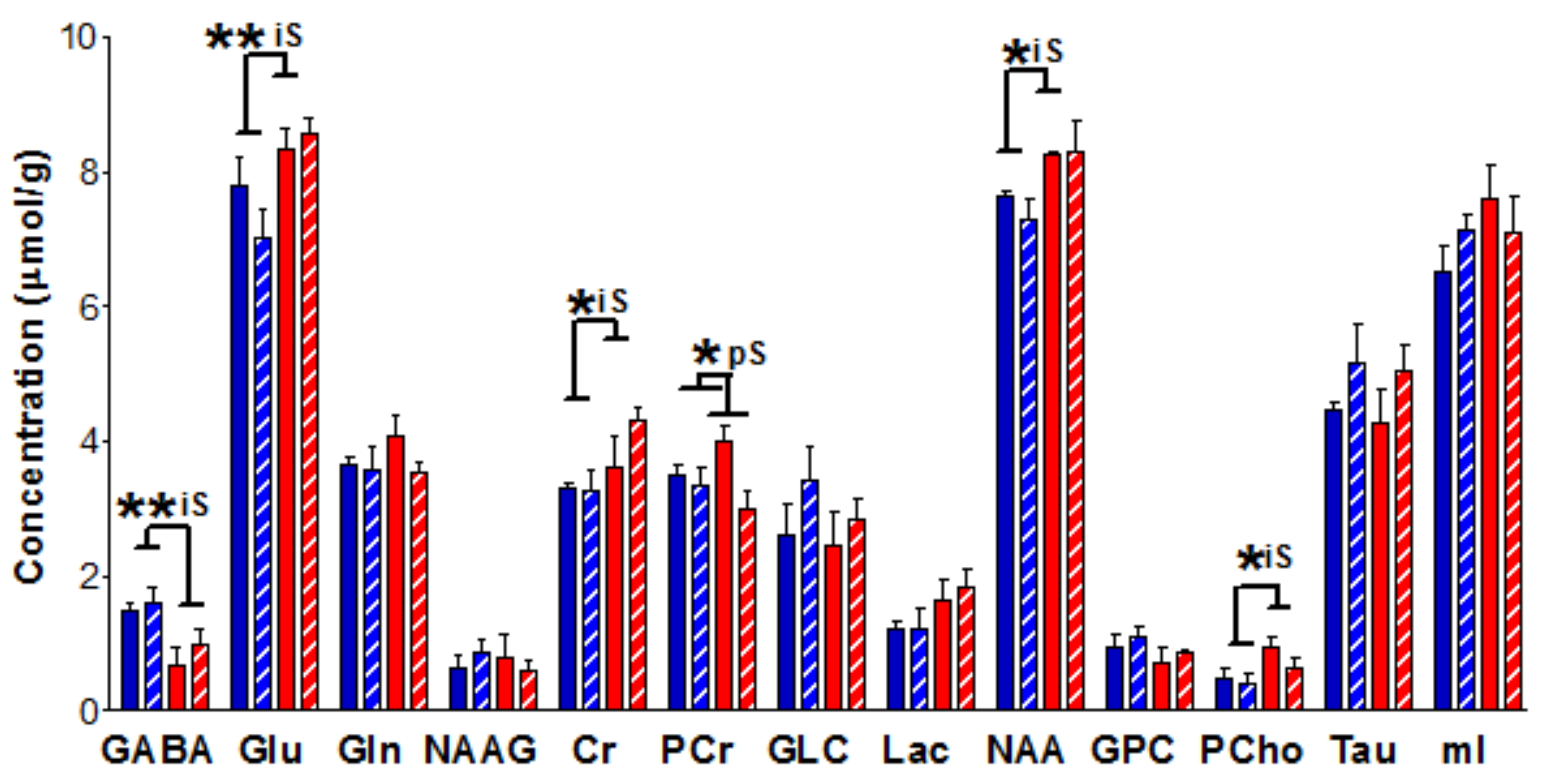

\section{$\square$ pC-ic $\quad \square p s$-ic $\square$ pC-is $\square$ ps-is}

Figure 3. Effects of paternal and individual peripubertal stress on the lateral septum neurochemical profile. The results are the mean + SEM. N=5-6/group. Two-way ANOVA between main effects: ${ }^{*}$, $P<0.05 ;{ }^{* *}, P<0.01$. 

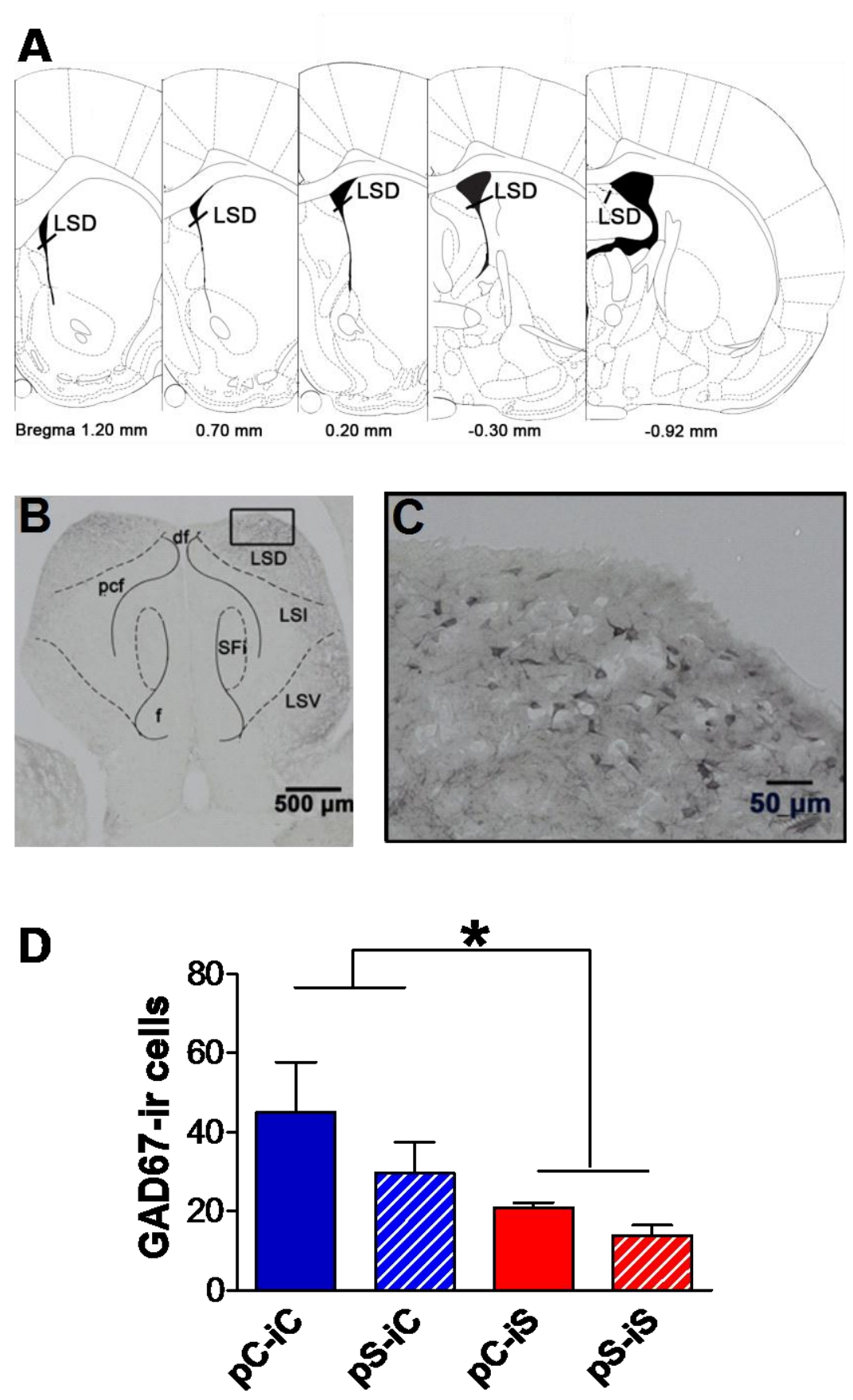

Figure 4. GAD67 immunohistochemistry of the dorsal lateral septum (LSD). Drawing [A, adapted from the Paxinos and Watson atlas (37)] depicts the rostrocaudal planes of the area of interest (Bregma range from $1.20 \mathrm{~mm}$ to $-0.92 \mathrm{~mm}$ ). Brightfield photomicrograph showing GAD67-ir within the LSD (B). Higher magnification of the boxed region is presented in (C). Comparison between groups of the density of 
GAD67-ir cells in the dorsal lateral septum (D), the results are the mean + SEM. Abbreviations: df, dorsal fornix; f, fornix; LSD, dorsal lateral septum; LSI, intermediate part of the lateral septum; LSV, ventral lateral septum; pcf, precommissural fornix; SFi, septofimbrial nucleus. Scale bar $=500 \mu \mathrm{m}$, boxed region= $50 \mu \mathrm{m} . N=5-6 /$ group. Two-way ANOVA on the logarithmic transformed measures: iS: ${ }^{*}, P<0.05$. 